\title{
"Fountain of Steroid Deep Within" A Case Report of an Ectopic ACTH-producing Tumor
}

Tom Edward N. Lo M.D., Imelda Antonio M.D. and Cecilia Jimeno M.D.

University of the Philippines- Philippine General Hospital- Department of Medicine

\section{ABSTRACT}

This paper reports a case of a young female suddenly presenting with features of hypercortisolism later on being discovered to be from an ectopic tumor producing elevated levels of ACTH leading to complications and eventually death of patient. On autopsy, it was discovered that the ectopic tumor was from a neuroendocrine tumor found at the pancreatic head with multiple metastatic lesions to the liver.

\section{BACKGROUND AND SIGNIFICANCE}

Cushing's Syndrome defined as a hormonal disorder resulting from elevated serum cortisol leading to specific physical and biochemical abnormalities that might be detrimental to life itself. Majority is caused by steroid intake while endogenous sources although representing only a minority of patients often lead to delay in diagnosis. Knowing their rare presentations and diagnostic clues might help physicians in the future to easily diagnose and manage endogenous Cushing's syndrome since most are completely reversible. The association of Cushing's syndrome with $\mathrm{ACTH}$-secreting tumors of non-pituitary and nonadrenal origin has been referred as Ectopic ACTHproducing tumor.

\section{HISTORY AND PHYSICAL EXAMINATION}

A previously healthy 39 year-old Filipino female is presented with a rapid development of features compatible with Cushing's syndrome (truncal obesity, buffalo hump, acne formation, moon facies, plethora, muscle wasting, acanthosis nigricans) in a span of 2 months. She also presented with metabolic alkalosis and significant hypokalemia with proximal muscle weakness unresponsive to potassium correction. There was also an associated hyperpigmentation of peculiar body sites (knuckles and toes). Patient eventually referred for acutely worsening behavioural and psychiatric changes.

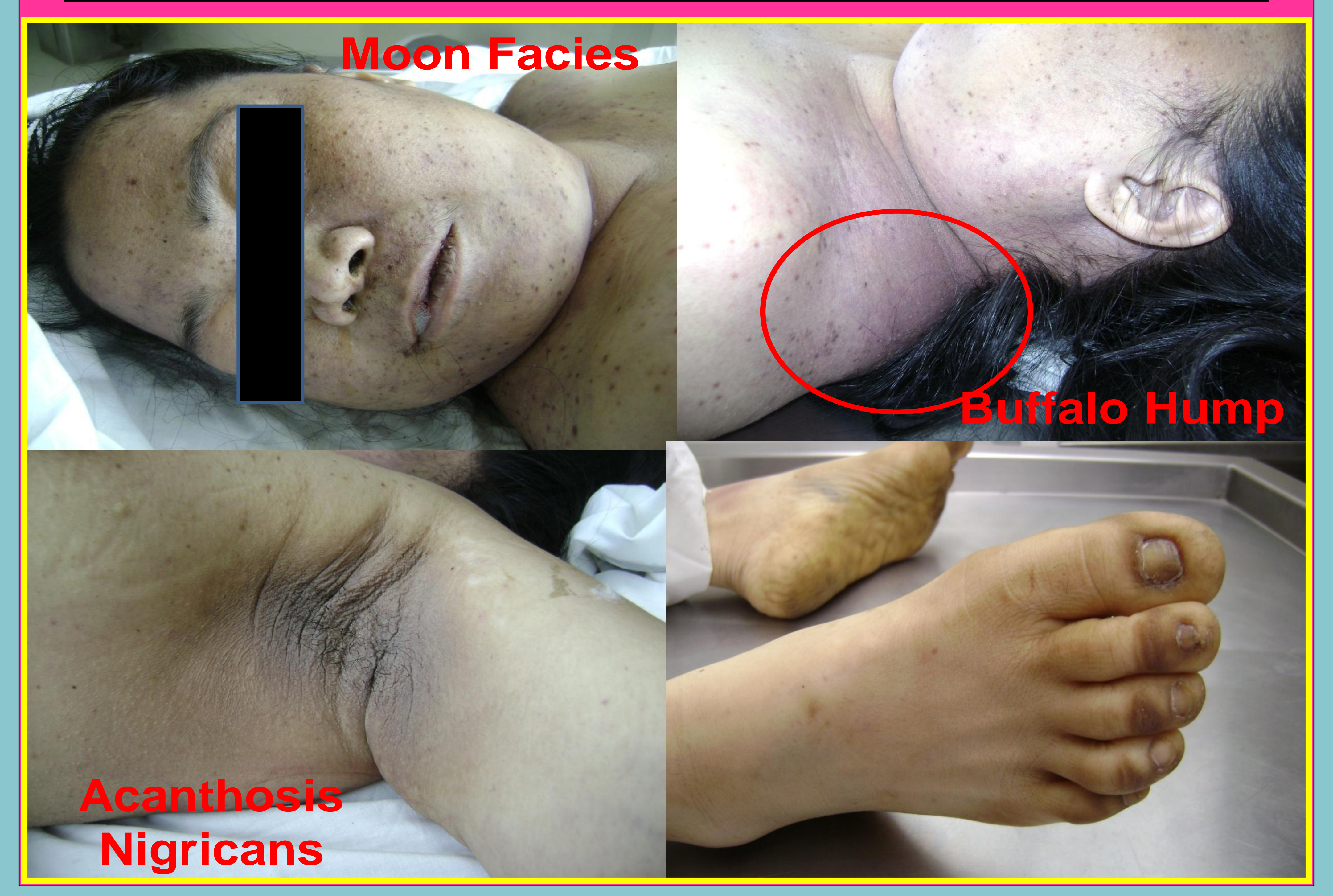

\section{DIAGNOSTIC WORK-UP}

With further work ups and repeated hospitalizations, insulin resistance (secondary diabetes), hypertension, dyslipidemia and thyroid function abnormalities (Low FT3, Normal FT4, Low TSH) were noted. Patient was also worked up for Endogenous Cushing's Syndrome with 12x elevated Serum Cortisol and a positive Dexamethasone suppression test. Due to the rapid clinical course, an ectopic source of steroid function was suspected. Elevated serum ACTH level strengthened the possibility of an Ectopic ACTH producing tumor.

Further work ups were requested to locate the tumor but patient eventually succumbed to sepsis from nosocomial infection. On autopsy, an ectopic well differentiated neuroendocrine tumor was found at the pancreatic head with metastasis to the right hepatic lobe.

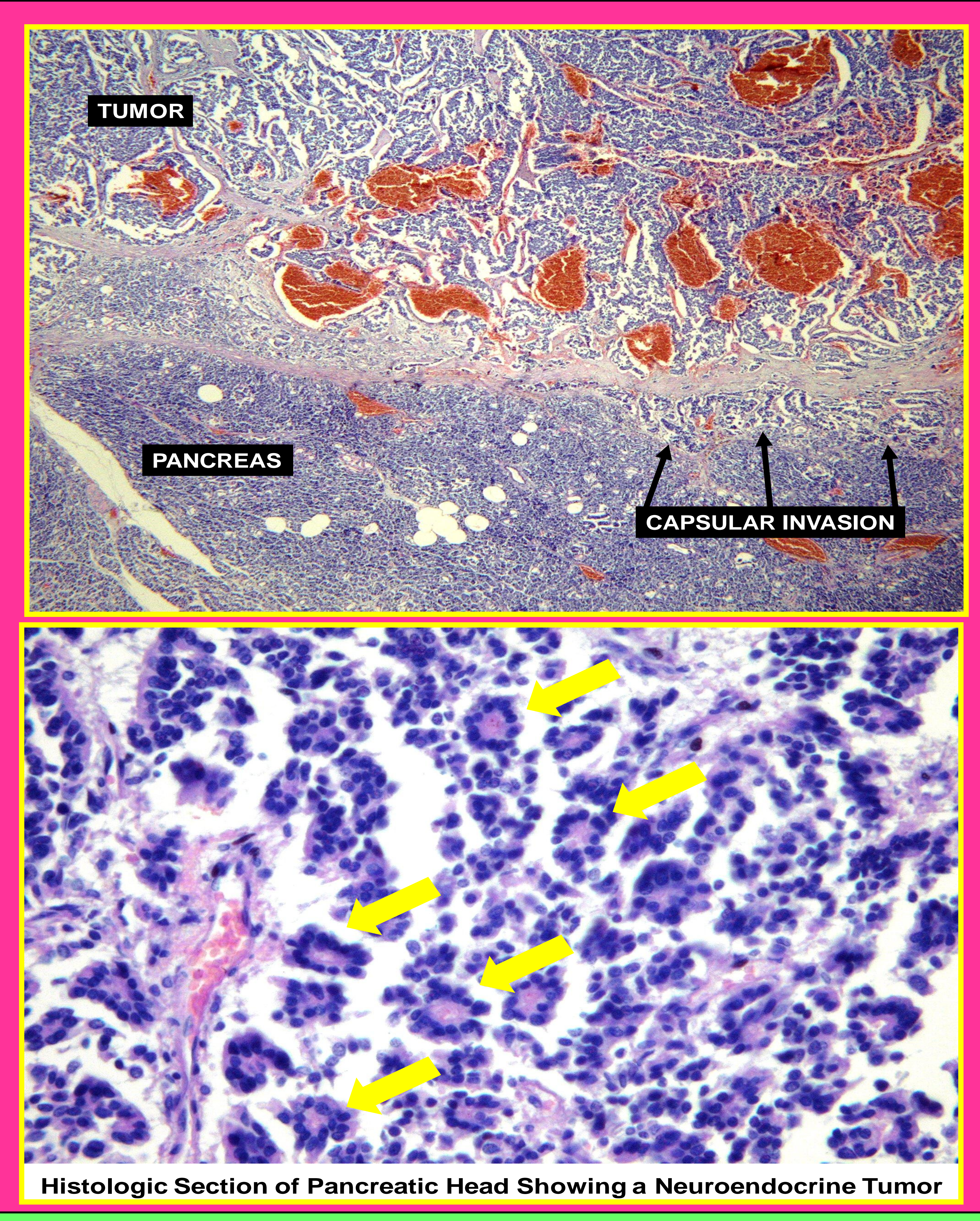

\section{RECOMMENDATION}

This case report highlights a rare case of ectopic ACTH syndrome and discusses its clinical manifestations and laboratory findings. Early detection of tumor site and possible removal is of utmost importance in managing these patients. Clinical diagnosis together with diagnostic algorithm can help and guide physicians in effectively diagnosing patients presenting with endogenous Cushing's syndrome. Finally, Lung, Gl tract and pancreas remains to be the usual site although unique and peculiar sites like prostate, esophagus and larynx might be a possibility. 